\title{
Evolution of the clinical presentation of men undergoing radical prostatectomy for high-risk prostate cancer
}

\author{
Phillip M. Pierorazio, Ashley E. Ross, Misop Han, Jonathan I. Epstein, Alan W. Partin, and \\ Edward M. Schaeffer \\ Brady Urological Institute, Johns Hopkins University, Baltimore, MD, USA
}

\section{Abstract}

- To investigate the outcomes and potential effect of improved longitudinal screening in men presenting with high-risk (advanced clinical stage [ $>\mathrm{T} 2 \mathrm{~b}$ ], Gleason score 8-10 or prostate-specific antigen [PSA] level $>20 \mathrm{ng} / \mathrm{mL}$ ) prostate cancer (PC).

- The Institutional Review Board approved, Institutional Radical Prostatectomy Database (1992-2010) was queried for men with high-risk PC based on D'Amico criteria.

- Year of surgery was divided into two cohorts: the Early PSA Era (EPE, 1992-2000) and the Contemporary PSA Era (CPE, 2001-2010).

- $\quad \mathrm{PC}$ features and outcomes were evaluated using appropriate comparative tests.

- In total, 667 men had high-risk PC in the EPE and 764 in the CPE.

- In the EPE, 598 (89.7\%) men presented with one high-risk feature; 173 (29.0\%) men had a Gleason score of 8-10 on biopsy. In the CPE, 717 (93.9\%) men presented with one high-risk feature $(P=0.004)$ and $494(68.9 \%)$ men had a Gleason score of 8-10.

- At 10 years, biochemical-free survival (BFS) was $44.1 \%$ and $36.4 \%$ in the EPE and CPE, respectively $(P=0.04)$; metastases-free survival (MFS) was $77.1 \%$ and $85.1 \%(P=0.6)$; and PC-specific survival (CSS) was $83.3 \%$ and $96.2 \%(P=0.5)$.

- BFS, MFS and CSS were worse for men with more than one high-risk feature in both eras.

- Over the PSA era, an increasing percentage of men with high-risk PC were categorized by a biopsy Gleason score of 8-10.

- The accumulation of multiple high-risk features increases the risk of biochemical recurrence, the development of metastases and death from PC.

- BFS, MFS and CSS are stable over the PSA era for these men. The balance between a greater proportion of men having high Gleason disease and a greater proportion with small, less advanced tumours may explain the stability in MFS and CSS over time.

\section{Keywords}

high-risk; outcomes; prostate cancer

Correspondence to Phillip M. Pierorazio, Johns Hopkins University - Urology, 600 N Wolfe Street Baltimore, MD 21287, USA. philpierorazio@jhmi.edu. 


\section{Introduction}

Developed in the late 1990s, the D'Amico criteria use clinical stage, serum PSA level and Gleason score to stratify men into low-, intermediate- and high-risk prostate cancer (PC) groups [1]. These criteria have been modified and validated to predict outcome after surgery or radiation [2]. According to these criteria, men with high-risk PC present with at least one of the criteria: advanced clinical stage (> T2b), Gleason score $8-10$ or PSA > $20 \mathrm{ng} / \mathrm{mL}$. The implementation of widespread PSA screening has changed the clinical presentation of men with PC, making it more probable that men present with a lower clinical stage and PSA level [3-5]. Subsequently, the proportion of men presenting with high-risk PC has decreased; falling from $40 \%$ to $15 \%$ in at least one large, population-based study [6]. We hypothesize that, over the recent PSA era, men with improved longitudinal screening would present with different high-risk features and potentially have improved outcomes. Therefore, the present study examined a large, institutional radical prostatectomy (RP) database for trends in highrisk disease presentation and outcomes.

\section{Patients and methods}

The Institutional Review Board approved, Johns Hopkins Radical Prostatectomy Database was queried from 1992 to 2010 for men with high-risk PC based on D'Amico criteria [1]. Year of surgery was divided into two cohorts: the Early PSA Era (EPE, 1992-2000) and the Contemporary PSA Era (CPE, 2001-2010). Presenting features and pathological outcomes were evaluated among eras with appropriate comparative tests ( $t$-test, chi-squared, ANOVA). The Kaplan-Meier method with log-rank test was used to determine biochemical-free (BFS), metastases-free (MFS) and cancer-specific survival (CSS). Proportional hazards modelling was used to predict outcomes of BFS, MFS and CSS in men with one and multiple high-risk features.

\section{Results}

Of the more than 19000 men treated with RP, 1431 were identified with high-risk PC from 1992 to 2010. Of those, 667 and 764 men underwent RP in the EPE and CPE, respectively, representing $10.6 \%$ and $6.9 \%$ of the entire RP population $(P<0.001)$. The presenting features are shown in Table 1. Of note, men in the CPE were clinically low-risk $(\leq \mathrm{cT} 2 \mathrm{a})$ almost $75 \%$ of the time, had a lower mean PSA level, $70 \%$ had a Gleason score of 8-10 at biopsy, 50\% had a Gleason score of 8-10 at final pathology, and tumours were more often organ-confined (pT2) at RP. Additionally, although men in the EPE had a higher mean PSA level and more advanced clinical and pathological stage, they had a lower Gleason sum at biopsy at final pathological analysis.

In the EPE, 598 (89.7\%) men presented with a single high-risk feature; of those, 219 (36.7\%) men had a higher PSA level, $173(29.0 \%)$ men had a Gleason score of 8-10 on biopsy and $206(34.5 \%)$ men were > cT2b. In the CPE, 717 (93.9\%) men presented with a single high-risk feature $(P=0.004)$; of those, $139(19.7 \%)$ men had a higher PSA level, 494 $(68.9 \%)$ men had a Gleason score of $8-10$ and $84(12.5 \%)$ men were $>$ cT2b $(P=0.01)$. The proportion of men presenting with specific high-risk features and the evolution of these features are shown in Fig. 1. Of note, a high-risk Gleason sum accounted for almost 20\% of the high-risk features in 1992 and $>75 \%$ by 2010.

For men with high-risk PC, 10-year BFS was $44.1 \%$ and $36.4 \%$ in the EPE and CPE, respectively $(P=0.04)$; 10 -year MFS was $77.1 \%$ and $85.1 \%(P=0.6)$; and 10-year CSS was $83.3 \%$ and $96.2 \%(P=0.5)$. In the EPE, 10 -year BFS was $47.5 \%$ vs $15.1 \%$ for men with one $(n=598 ; 89.7 \%)$ and more than one $(n=69 ; 10.4 \%)$ high-risk feature, respectively $(P<$ 
$0.001)$; 10-year MFS was $80.1 \%$ and $50.9 \%(P<0.001)$; and 10 -year CSS was $85.4 \%$ and $67.8 \%(P<0.001)$. In the CPE, 10 -year BFS was $37.9 \%$ for men with one high-risk feature $(n=717 ; 93.9 \%)$ and $23.8 \%$ at 4 years for men with more than one high-risk feature $(n=47$; $6.2 \%)$, respectively $(P<0.001)$; 10 -year MFS was $85.7 \%$ and $74.1 \%(P<0.009)$; and 10 year CSS was $96.5 \%$ and $92.3 \%(P=0.3)$. Kaplan-Meier curves for BFS, MFS and CSS survival by era are shown in Fig. 2. In the study cohort, regression analyses showed the hazard ratios for men with more than one high-risk feature to be 2.08 (95\% CI, 1.48-2.9; $P$ $<0.001)$ and $2.6(95 \% \mathrm{CI}, 1.58-4.2 ; P<0.001)$ for biochemical recurrence in the EPE and CPE, respectively. For metastatic recurrence, the hazard ratios were 2.3 (95\% CI, 1.38-3.9; $P=0.001)$ and $3.8(95 \% \mathrm{CI}, 1.28-11.4 ; P=0.02)$ for the EPE and CPE, respectively. For PC-associated mortality, the hazard ratios were 2.7 (95\% CI, 1.66-4.5; $P<0.001)$ and 2.9 (95\% CI, 0.33-24.6; $P=0.4$ ) for the EPE and CPE, respectively. Multivariable regression analyses predicting biochemical recurrence, metastatic recurrence and PC specific mortality are shown in Table 2. A high-risk Gleason sum was associated with an 3.6-, 5.4- and 8.6fold greater risk of biochemical recurrence, metastatic recurrence and PC mortality, respectively.

In total, 307 (34.4\%) men received additional adjuvant or salvage therapy after RP. There were $118(13.3 \%)$ men who received adjuvant therapy (within the first year after RP). Of those, $39(33.1 \%)$ men received androgen deprivation, 27 (22.9\%) men received radiation therapy and $45(38.1 \%)$ men received combined androgen deprivation and radiation therapy.

\section{Discussion}

PSA screening has increased the detection of PC and reduced PC-specific mortality [7]. Screening has also shifted the stage at presentation for men with PC, including men with high-risk disease who now present with less advanced disease [6]. This analysis describes the evolution of high-risk PC in a large surgical series and, additionally, describes the longterm oncological outcomes for men in the Early and Contemporary PSA Eras. Taken together, these findings provide a better understanding of the factors associated with improved outcomes for these men.

Although it is established that men with high-risk PC experience recurrence, metastases and death at a highest rate in the $\mathrm{PC}$ population; the data from the present study show a shift toward improved preoperative clinical characteristics for men with high-risk PC, with fewer presenting with multiple high-risk features and less with clinically advanced disease.

Nevertheless, MFS and CSS are statistically equivalent across the EPE and CPE eras. This phenomenon has been reported in other studies $[8,9]$ and we consider that this dichotomy can be explained by a number a balancing factors. This may simply reflect differences in follow-up because, by definition, the EPE has a longer exposure time for all outcomes. Accordingly, the finding that MFS and CSS are not statistically improved in the present analysis suggests a trend toward improved outcomes, which may reach statistical significance with longer follow-up. Other studies have alluded to this phenomenon, showing that PC outcomes are a moving target, with updates of nomograms and predictive algorithms showing not only improvements in outcomes based both on a shift toward more favourable clinical characteristics, but also improvements within certain risk strata $[6,10]$. Therefore, including the most recent data from our large, comprehensive database improves our understanding of the current outcomes for high-risk PC and enhances our ability to predict future outcomes. Alternatively, the improved clinical characteristics may reflect trends in surgeon selection bias because urologists intuitively chose to operate on those men who appear to be most curable by preoperative parameters. 
The most notable evolution in the presentation of men with high-risk PC is the shift toward high-risk Gleason predominant features. In our series, before 2001, high-risk Gleason as a solitary feature constituted less than $40 \%$ of the RP population per year and, subsequent to 2004 , at least $60 \%$ of the RP population per year. This most certainly reflects improvements in longitudinal PSA screening in the CPE, leading to fewer patients with higher PSA levels and less men with locally advanced PC. Stated differently, longitudinal PSA screening may improve tumour stage and PSA risk through early detection, although not necessarily changing the biology and prevalence of high-grade PC. This finding is paralleled in a study by Kane et al. [8] where the rates designation of being at high-risk by Gleason score increased from $23 \%$ to $68 \%$ between 1988 and 2003. It must be acknowledged that these are surgical series and changes in presenting features may also represent shifts in surgeon selection criteria and bias. However, the results from the CaPSURE, a non-surgery specific database, showed similar shifts in the defining characteristics of men with high-risk PC [6], although those data were current only through 2002 and the series of the present study extends the data up to 2010. An additional consequence of improved PC screening would be a shift toward lower volume cancers. Coupled with the standard use of extended, 12-core biopsy templates [11], it a plausible to hypothesize that, although more men went to RP with a high-risk Gleason sum, these tumours were smaller and probably organ-confined. Although no study has specifically addressed tumour volume in high-risk patients (or high Gleason disease), substantial data have shown a shift toward smaller volume, early stage tumours in the contemporary era of improved longitudinal PSA screening and 12-core biopsies [12-14]. Therefore, the balance between a greater proportion of men having high Gleason disease and a greater proportion with small, less advanced tumours may explain the stability in MFS and CSS over time.

It is important to recognize that grade migration and the Will Rogers phenomenon may also account for some of the apparent changes in high-risk presentation and outcomes [15]. Grade migration most strongly refers to older Gleason score 7 tumours now classified as Gleason score 8 and certainly exists. However, no data have clearly shown that new Gleason score 8 cancers are less aggressive than old Gleason score 8 cancers. Indeed, the current literature, including the present series, indicates that outcomes for a Gleason score of 8-10 are equivalent during the past few decades $[8,9,15]$. Therefore, the Will Rogers phenomenon is relevant in comparing Gleason score 7 cancers in the old and contemporary classification schemes, and should have little bearing on the analysis in the present study. An additional important point to be gleaned from the data obtained in the present study is that the accumulation of high-risk features has a poor prognosis. Intuitively, a higher PSA level and advanced clinical stage indicate larger, potentially locally advanced tumours. In the EPE, men with more than one high-risk feature had drastically different rates of BFS, MFS and CSS. The magnitude of difference in BFS, MFS and CSS is less in the CPE, again probably reflecting differences in the length of follow-up after RP and the presenting characteristics of these men. The accumulation of high-risk features has been shown to predict adverse outcomes after radiation treatment [16,17]; however, outcomes after RP have not been clearly elucidated. From the data obtained in the present study and those of others, it is clear that the Gleason score drives most of the outcomes after RP, as is evident by the largest hazard ratios in multivariable analysis. For example, in the multivariable analysis of the present study, the hazard ratios for high-risk Gleason sum are one to three times the order of magnitude for an increased risk of BFS, MFS and CSS. The addition of multiple high-risk features to the Gleason sum can therefore have drastic influences on prognosis. For example, a patient with a Gleason score of 8 at biopsy as their only high-risk feature has a 3.6-fold higher risk of biochemical recurrence, a 5.4-fold higher risk of metastases and an 8.6-fold higher risk of PC mortality according to the data obtained in the present study. If the same patient had an abnormal DRE (> clinical T2b), the subsequent risk of biochemical 
recurrence, metastatic recurrence and mortality would rise to 5.2-, 7.6- and 11.4-fold, respectively.

These findings, together with previous research, may help in the consultation and selection of high-risk PC patients for RP. Many patients with high-risk PC undergo external beam radiation therapy in favour of RP $[18,19]$. The data obtained in the present study indicate that men with a solitary high-risk feature, most commonly a Gleason score of 8-10, have favourable outcomes after RP. Although those with one high-risk feature have the best prognosis, patients with multiple features may benefit from surgery as part of a multimodal therapy compared to radiation therapy, as indicated by another recent study [20]. Although it is considered that nerve-sparing may be more challenging and positive surgical margin rates may be higher in more advanced disease, high-risk patients should not be excluded from treatment algorithms that include radical surgery when surgical series show a similar duration of recovery, continence, complications and perioperative morbidity among patients undergoing RP with high- and low-risk features [21,22]. A previous study from our institution regarding patients with a Gleason score of 8-10 shows that, although upwards of $40 \%$ of men with high-risk PC with require additional adjuvant or salvage therapy, men with pT2 or pT3a disease can expect a 15-year CSS of 80-90\% [23].

Many of the obvious limitations of the present study have been discussed previously, including the inherent bias of a post-surgical series and the difference in follow-up times inherent in analyzing different time periods. The database of the present study is expertly maintained in a prospective fashion, ensuring a thorough follow-up of adequate length for both groups. Additionally, the use and timing of adjuvant and salvage therapies may help guide the therapeutic algorithm for the treatment of men with high-risk PC. It is the practice pattern at our institution to use adjuvant radiation only for those patients with positive surgical margins without seminal vesicle or lymph node invasion, and only to administer androgen deprivation at the appearance of radiographically-evident or symptomatic metastases because immediate hormone treatment has not been shown to improve overall survival [24-27]. Because these patients are have adverse PC findings, gleaning meaningful results from the present study cohort regarding the use of adjuvant and salvage therapies is difficult. Previous studies involving this cohort have discussed these results [23] and the focus of the present study was to use preoperative parameters to describe the changing face of high-risk PC, aiming to better select and counsel patients regarding the use of RP in the treatment of their disease.

In conclusion, over the PSA era, an increasing percentage of men with high-risk PC were classified as high-risk based on a biopsy Gleason score of 8-10, possibly reflecting the earlier detection high-grade lesions or changes in PC grading. Nevertheless, BFS, MFS and CSS are stable over the PSA era for men with high-risk PC. The accumulation of multiple high-risk features increases the risk of biochemical recurrence, development of metastases and death from PC.

\section{Acknowledgments}

The content is solely the responsibility of the authors and does not necessarily represent the official views of the National Institute of Diabetes and Digestive and Kidney Diseases or the National Institutes of Health.

Conflict of interest This study was supported by SPORE grant P50CA58236 from the National Institutes of Health and the National Cancer Institute. Phillip Pierorazio and Ashley Ross are supported by award Number T32DK007552 from the National Institute of Diabetes and Digestive and Kidney Diseases. 


\section{Abbreviations}

$\begin{array}{ll}\text { BFS } & \text { biochemical-free survival } \\ \text { CPE } & \text { Contemporary PSA Era } \\ \text { CSS } & \text { cancer-specific survival; metastases-free survival } \\ \text { EPE } & \text { Early PSA Era } \\ \text { PS } & \text { prostate cancer } \\ \text { RP } & \text { radical prostatectomy }\end{array}$

\section{References}

1. D'Amico AV, Whittington R, Malkowicz SB, et al. Biochemical outcome after radical prostatectomy, external beam radiation therapy, or interstitial radiation therapy for clinically localized prostate cancer. JAMA. 1998; 280:969. [PubMed: 9749478]

2. McAleer SJ, Schultz D, Whittington R, et al. PSA outcome following radical prostatectomy for patients with localized prostate cancer stratified by prostatectomy findings and the preoperative PSA level. Urol Oncol. 2005; 23:311. [PubMed: 16144663]

3. Moul JW, Wu H, Sun L, et al. Epidemiology of radical prostatectomy for localized prostate cancer in the era of prostate-specific antigen: an overview of the Department of Defense Center for Prostate Disease Research national database. Surgery. 2002; 132:213. [PubMed: 12219014]

4. Ung JO, Richie JP, Chen MH, et al. Evolution of the presentation and pathologic and biochemical outcomes after radical prostatectomy for patients with clinically localized prostate cancer diagnosed during the PSA era. Urology. 2002; 60:458. [PubMed: 12350484]

5. Jani AB, Vaida F, Hanks G, et al. Changing face and different countenances of prostate cancer: racial and geographic differences in prostate-specific antigen (PSA), stage, and grade trends in the PSA era. Int J Cancer. 2001; 96:363. [PubMed: 11745507]

6. Cooperberg MR, Lubeck DP, Mehta SS, et al. Time trends in clinical risk stratification for prostate cancer: implications for outcomes (data from CaPSURE). J Urol. 2003; 170:S21. [PubMed: 14610406]

7. Schroder FH, Hugosson J, Roobol MJ, et al. Screening and prostate-cancer mortality in a randomized European study. N Engl J Med. 2009; 360:1320. [PubMed: 19297566]

8. Kane CJ, Presti JC Jr, Amling CL, et al. Changing nature of high risk patients undergoing radical prostatectomy. J Urol. 2007; 177:113. [PubMed: 17162017]

9. Boorjian SA, Karnes RJ, Rangel LJ, et al. Impact of prostate-specific antigen testing on the clinical and pathological outcomes after radical prostatectomy for Gleason 8-10 cancers. BJU Int. 2008; 101:299. [PubMed: 17922854]

10. Makarov DV, Trock BJ, Humphreys EB, et al. Updated nomogram to predict pathologic stage of prostate cancer given prostate-specific antigen level, clinical stage, and biopsy Gleason score (Partin tables) based on cases from 2000 to 2005. Urology. 2007; 69:1095. [PubMed: 17572194]

11. Terris MK. Prostate biopsy strategies: past, present, and future. Urol Clin North Am. 2002; 29:205. [PubMed: 12109346]

12. Iczkowski KA, Hossain D, Torkko KC, et al. Preoperative prediction of unifocal, unilateral, margin-negative, and small volume prostate cancer. Urology. 2008; 71:1166. [PubMed: 18279927]

13. Cooperberg MR, Lubeck DP, Meng MV, et al. The changing face of low-risk prostate cancer: trends in clinical presentation and primary management. J Clin Oncol. 2004; 22:2141. [PubMed: 15169800]

14. Polascik TJ, Mayes JM, Sun L, et al. Pathologic stage T2a and T2b prostate cancer in the recent prostate-specific antigen era: implications for unilateral ablative therapy. Prostate. 2008; 68:1380. [PubMed: 18543281]

15. Albertsen PC, Hanley JA, Barrows GH, et al. Prostate cancer and the Will Rogers phenomenon. J Natl Cancer Inst. 2005; 97:1248. [PubMed: 16145045] 
16. D’Amico AV, Chen MH, Catalona WJ, et al. Prostate cancer-specific mortality after radical prostatectomy or external beam radiation therapy in men with 1 or more high-risk factors. Cancer. 2007; 110:56. [PubMed: 17530618]

17. Nguyen PL, Chen MH, Catalona WJ, et al. Predicting prostate cancer mortality among men with intermediate to high-risk disease and multiple unfavorable risk factors. Int J Radiat Oncol Biol Phys. 2009; 73:659. [PubMed: 18692327]

18. Meng MV, Elkin EP, Latini DM, et al. Treatment of patients with high risk localized prostate cancer: results from cancer of the prostate strategic urological research endeavor (CaPSURE). $J$ Urol. 2005; 173:1557. [PubMed: 15821485]

19. Denberg TD, Glode LM, Steiner JF, et al. Trends and predictors of aggressive therapy for clinical locally advanced prostate carcinoma. BJU Int. 2006; 98:335. [PubMed: 16879674]

20. Zelefsky MJ, Eastham JA, Cronin AM, et al. Metastasis after radical prostatectomy or external beam radiotherapy for patients with clinically localized prostate cancer: a comparison of clinical cohorts adjusted for case mix. J Clin Oncol. 2010; 28:1508. [PubMed: 20159826]

21. Berglund RK, Jones JS, Ulchaker JC, et al. Radical prostatectomy as primary treatment modality for locally advanced prostate cancer: a prospective analysis. Urology. 2006; 67:1253. [PubMed: 16678888]

22. Loeb S, Smith ND, Roehl KA, et al. Intermediate-term potency, continence, and survival outcomes of radical prostatectomy for clinically high-risk or locally advanced prostate cancer. Urology. 2007; 69:1170. [PubMed: 17572209]

23. Pierorazio PM, Guzzo TJ, Han M, et al. Long-term survival after radical prostatectomy for men with high gleason sum in pathologic specimen. Urology. 2010; 76:715. [PubMed: 20350749]

24. Van der Kwast TH, Bolla M, Van Poppel H, et al. Identification of patients with prostate cancer who benefit from immediate postoperative radiotherapy: EORTC 22911. J Clin Oncol. 2007; 25:4178. [PubMed: 17878474]

25. Wiegel T, Bottke D, Steiner U, et al. Phase III postoperative adjuvant radiotherapy after radical prostatectomy compared with radical prostatectomy alone in pT3 prostate cancer with postoperative undetectable prostate-specific antigen: ARO 96-02/AUO AP 09/95. J Clin Oncol. 2009; 27:2924. [PubMed: 19433689]

26. Loblaw DA, Virgo KS, Nam R, et al. Initial hormonal management of androgen-sensitive metastatic, recurrent, or progressive prostate cancer: 2006 update of an American Society of Clinical Oncology practice guideline. J Clin Oncol. 2007; 25:1596. [PubMed: 17404365]

27. Walsh PC. Urological Oncology: Prostate Cancer: Editorial Comment on: Intermittent Androgen Deprivation for Locally Advanced and Metastatic Prostate Cancer: Results From a Randomised Phase 3 Study of the South European Uroncological Group. J Urol. 2009; 182:2728. 


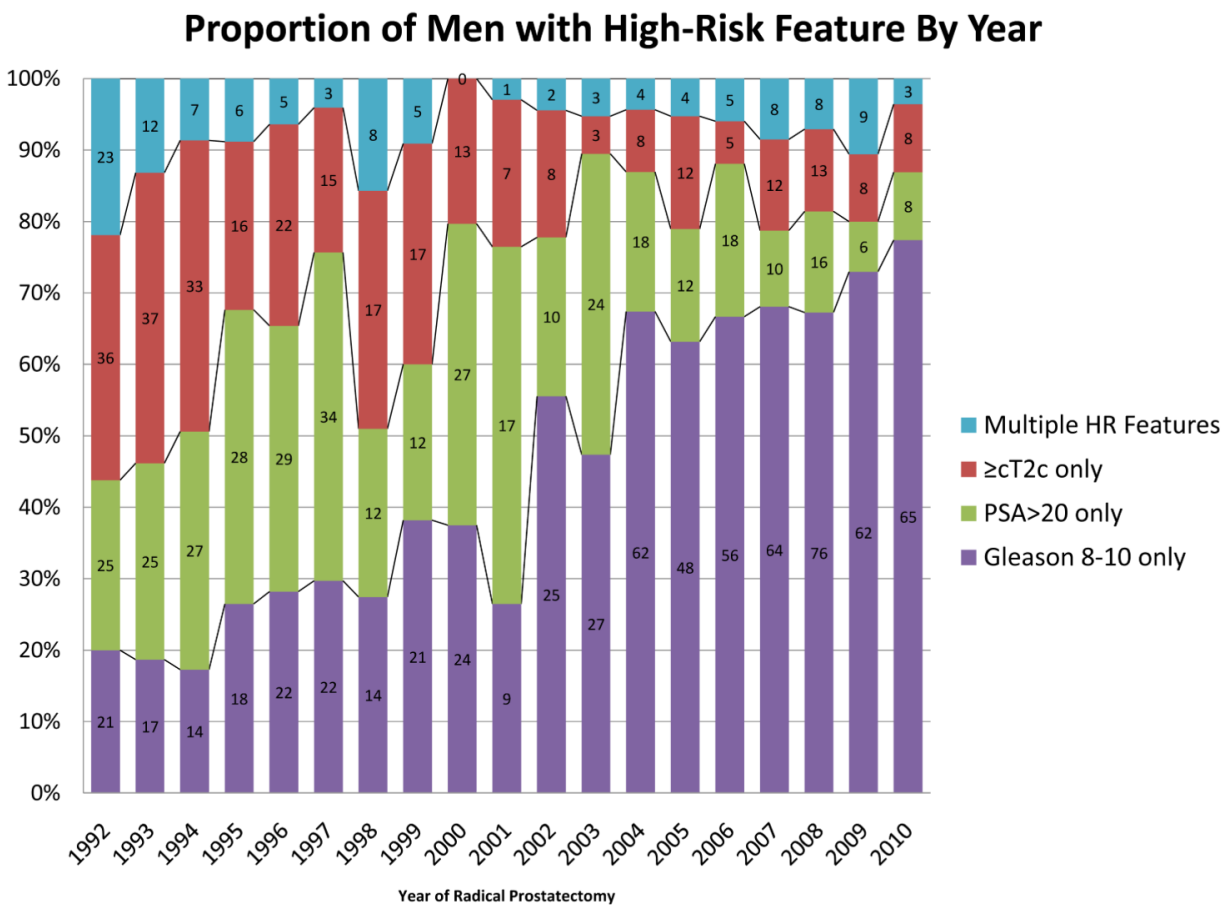

FIG. 1.

Trends in the presenting features of men with high-risk (HR) prostate cancer undergoing radical prostatectomy (1992-2010). 


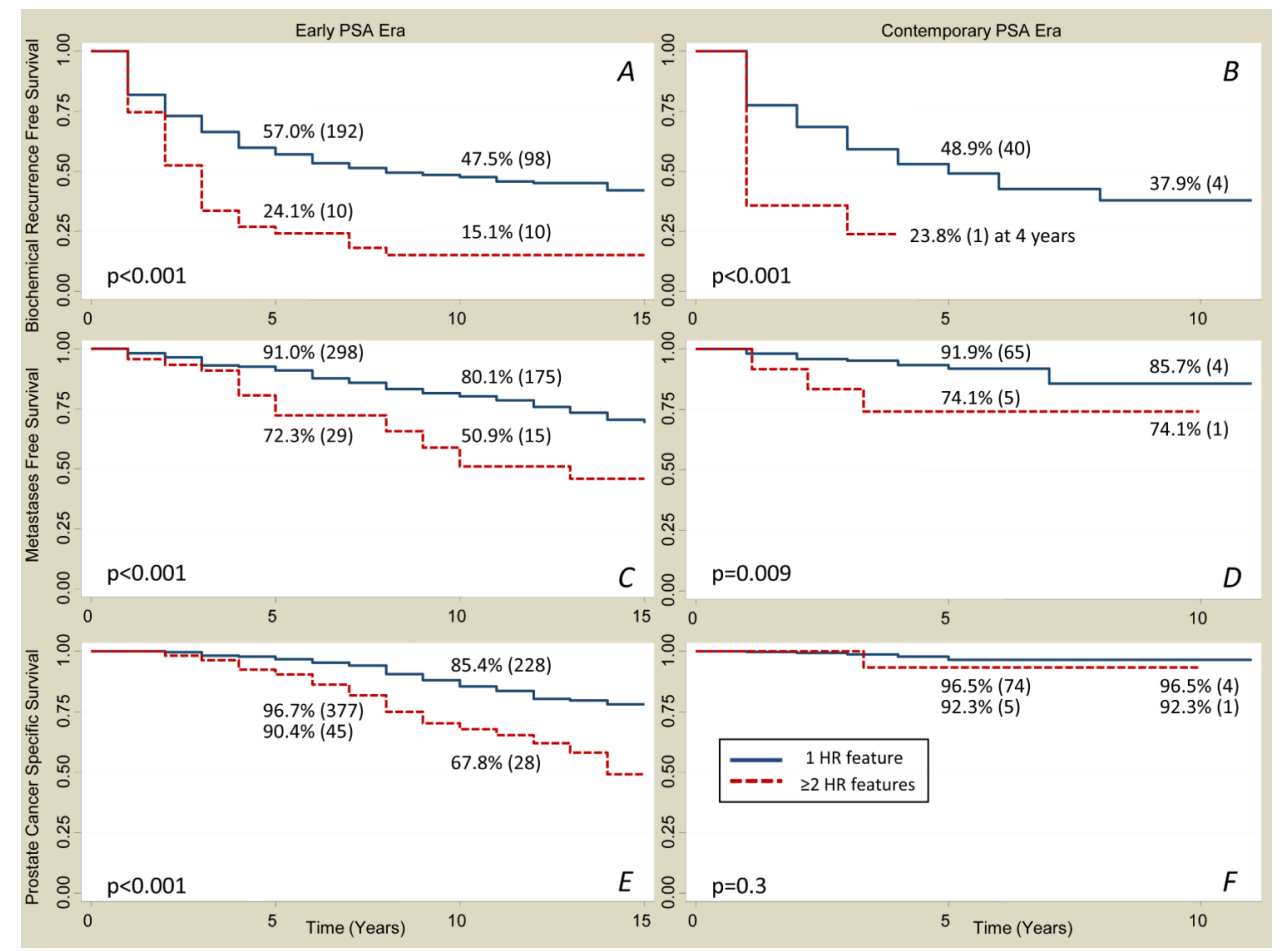

FIG. 2.

Survival outcomes for men with one and multiple features of high-risk (HR) PC in the Early PSA Era (EPE) and Contemporary PSA Era (CPE). Biochemical recurrence-free survival for EPE (A) and CPE (B); metastases-free survival for EPE (C) and CPE (D); and prostate cancer-specific survival for EPE (E) and CPE (F). Men with one high-risk feature are indicated by the solid line, those with more than one feature are indicated by the broken line. Proportion surviving is shown at 5 and 10 years; the number at risk at each time point is shown in parenthesis. 
TABLE 1

Preoperative characteristics of men with high-risk prostate cancer undergoing radical prostatectomy in the PSA era

\begin{tabular}{|c|c|c|c|}
\hline Variable & $\operatorname{EPE}(n=667)$ & $\mathrm{CPE}(n=764)$ & $P$ \\
\hline Age (years), median (range) & $59(37-73)$ & $59.5(38-76)$ & 0.19 \\
\hline \multicolumn{4}{|l|}{ Race, $n(\%)$} \\
\hline African American & $46(6.9)$ & $108(14.1)$ & $<0.001$ \\
\hline Asian & $1(0.1)$ & $6(0.8)$ & \\
\hline Caucasian & $598(89.7)$ & $614(80.4)$ & \\
\hline Other & $22(3.3)$ & $36(4.7)$ & \\
\hline Clinical stage, $n(\%)$ & & & $<0.001$ \\
\hline $\mathrm{cT} 1-\mathrm{cT} 2 \mathrm{a}$ & $318(47.7)$ & $536(72.3)$ & \\
\hline $\mathrm{cT} 2 \mathrm{~b}$ & $89(13.3)$ & $98(13.2)$ & \\
\hline $\mathrm{cT} 2 \mathrm{c}-\mathrm{cT} 3 \mathrm{~b}$ & $260(39.0)$ & $108(14.6)$ & \\
\hline PSA (ng/mL), median (range) & $17.4(0.1-116)$ & $11.9(0.2-97)$ & $<0.001$ \\
\hline Biopsy Gleason score, $n(\%)$ & & & $<0.001$ \\
\hline $2-6$ & $309(46.5)$ & $98(12.8)$ & \\
\hline 7 & $142(21.4)$ & $131(17.1)$ & \\
\hline $8-10$ & $214(32.2)$ & $535(70.0)$ & \\
\hline Pathological Gleason score, $n(\%)$ & & & $<0.001$ \\
\hline $2-6$ & $172(26.0)$ & $72(9.6)$ & \\
\hline 7 & $317(47.9)$ & $302(40.1)$ & \\
\hline $8-10$ & $173(26.1)$ & $379(50.3)$ & \\
\hline Pathological stage, $n(\%)$ & & & $<0.001$ \\
\hline pT2 & $184(27.7)$ & $304(39.8)$ & \\
\hline pT3a & $332(49.9)$ & 295 (38.6) & \\
\hline pT3b & $80(12.0)$ & $100(13.1)$ & \\
\hline N1 & $69(10.4)$ & $65(8.5)$ & \\
\hline
\end{tabular}

EPE, Early PSA Era; CPE, Contemporary PSA Era. 


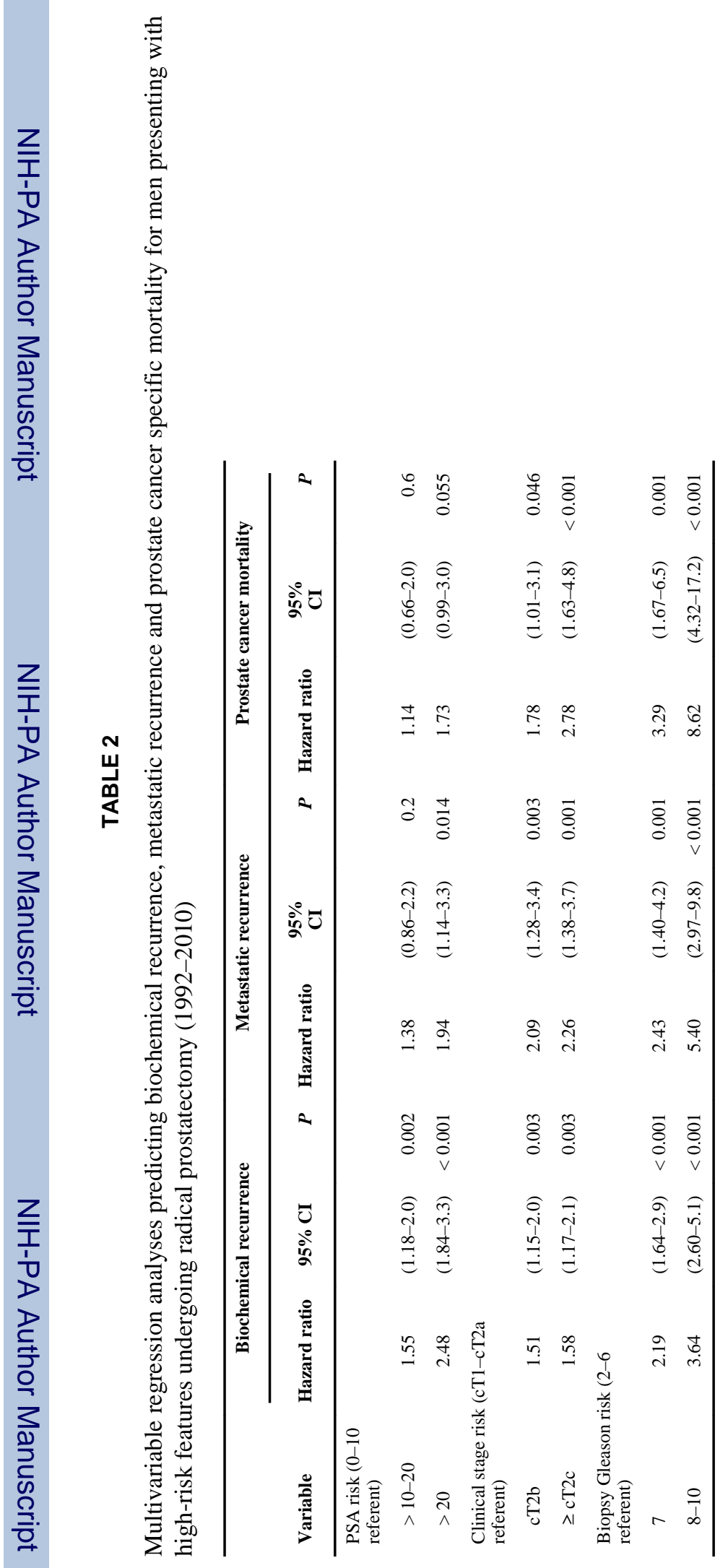

BJU Int. Author manuscript; available in PMC 2013 April 1. 